\title{
Elasticity of smectic liquid crystals with focal conic domains
}

\author{
S Fujiii ${ }^{1}$, S Komura ${ }^{2}$, Y Ishii ${ }^{3,5}$ and C-Y D Lu ${ }^{4}$ \\ ${ }^{1}$ Department of Chemistry, Nagaoka University of Technology, Niigata 940-2188, Japan \\ ${ }^{2}$ Department of Chemistry, Tokyo Metropolitan University, Tokyo 192-0397, Japan \\ ${ }^{3}$ Department of Organic and Polymeric Materials, Tokyo Institute of Technology, \\ Tokyo 152-8552, Japan \\ ${ }^{4}$ Department of Chemistry, National Taiwan University, Taipei 106, Taiwan \\ E-mail: sfujii@mst.nagaokaut.ac.jp
}

Received 1 February 2011, in final form 28 April 2011

Published 25 May 2011

Online at stacks.iop.org/JPhysCM/23/235105

\begin{abstract}
We study the elastic properties of thermotropic smectic liquid crystals with focal conic domains (FCDs). After the application of the controlled preshear at different temperatures, we independently measure the shear modulus $G^{\prime}$ and the FCD size $L$. We find out that these quantities are related by the scaling relation $G^{\prime} \approx \gamma_{\text {eff }} / L$, where $\gamma_{\text {eff }}$ is the effective surface tension of the FCDs. The experimentally obtained value of $\gamma_{\text {eff }}$ shows the same scaling as the effective surface tension of the layered systems $\sqrt{K B}$, where $K$ and $B$ are the bending modulus and the layer compression modulus, respectively. The similarity of this scaling relation to that of the surfactant onion phase suggests an universal rheological behavior of the layered systems with defects.
\end{abstract}

(Some figures in this article are in colour only in the electronic version)

\section{Introduction}

The rheology of smectic liquid crystals is a long-standing puzzle [1-3]. Although the theory predicts a liquid response when the smectic layers are aligned parallel to the shear plane, some experiments show that the system behaves as a solid until a certain critical shear stress is exceeded [4-6]. This means that even the oriented smectic liquid crystal behaves as a yield stress fluid at very low stress. Such a peculiar behavior can be attributed to the presence of defects such as focal conic domains (FCDs) in the smectic layers, because they act to hinder layer sliding in the low stress limit. Indeed it was seen that the FCD density strongly correlates with the viscoelastic properties [3, 4]. Previously we showed that the yield stress remarkably decreases around the SmA-N transition temperature and vanishes at the transition point [7]. These observations were attributed to the rapid increment of FCD size because the transition is induced by a smectic melting. It is known that the $\mathrm{SmA}-\mathrm{N}$ transition of $8 \mathrm{CB}$ is very close to the second-order one $[8,9]$. In general, softening of

\footnotetext{
5 Present address: Department of Physics, Kyoto University, Kyoto 606-8502, Japan.
}

the elasticity in the smectic close to the transition point can be induced by defect unbinding [10].

Prior to our work, it was reported that defects of oily streaks stabilized by colloidal particles reinforce the shear modulus which increases with the defect density [11, 12]. For the $8 \mathrm{CB}$ liquid crystal, it was shown that defects that are artificially introduced by the aerosil gel network result in the soft glassy nature of the smectic phase [13]. The shear modulus of the smectic phase is strongly influenced by the line tension of dislocations because it acts against the Peach-Koehler force which drives the motion of dislocations. The balance between these two forces determines the dislocation spacing which in turn affects the elasticity of the smectic phase. When the force exerted on the dislocations exceeds the line tension, the system undergoes plastic deformation. Meyer et al proposed a theory of smectic rheology associated with the motion of screw dislocations in the plastic deformation region [14, 15]. Using the Orowan relation [16], they predicted that the shear stress $\sigma$ and the shear rate $\dot{\gamma}$ follows the scaling relation $\sigma \sim \dot{\gamma}^{3 / 5}$ and the dislocation spacing $\xi$ decreases as $\xi \sim \dot{\gamma}^{-1 / 5}$. Hence the smaller $\xi$ results in the higher shear modulus.

On the other hand, it is known that the smectic phase is occupied by FCDs under shear. In thermotropic smectic 
liquid crystals without any additives, the proliferation of dislocation loops is induced by both equilibrium thermal fluctuations and non-equilibrium shear flow [17, 18]. Horn and Kleman investigated the origin of the elasticity of the smectic phase by comparing the rheometry and the birefringence measurements [4]. Based on the dimensional analysis, they proposed a scaling relation for the yield stress $\sigma_{\mathrm{y}}$ such that $\sigma_{\mathrm{y}} \sim K / L^{2}$, where $K$ is the bending modulus and $L$ is the FCD size. Although it is unknown how the dislocation unbinding affects the FCD size, the strong correlation between temperature and flow should be reflected in the shear modulus through the change in the FCD size.

The aim of this study is to investigate the origin of the elasticity in the smectic phase of a thermotropic liquid crystal (8CB) by comparing the shear modulus and the FCD size obtained from independent measurements. We discuss the preshear and the temperature dependences of the shear modulus, and relate it to the corresponding FCD size. The measured shear modulus is attributed to the energy cost for deformation of FCDs under shear. Thus their surface tension which acts against the deformation plays an important role in determining the shear modulus. The effective surface tension of the FCD is given by $\gamma_{\text {eff }}=\sqrt{K B}$, where $B$ is the compression modulus. Notice that $\gamma_{\text {eff }}$ has the dimension of energy per area. We demonstrate that the shear modulus is inversely proportional to the FCD size with the coefficient determined by the surface tension of FCDs. A notable analogy with the elasticity of the surfactant onion phase will also be discussed, thereby suggesting a universal rheological property of the layered structures with defects.

In our previous study on the same system, we showed that the two different types of layer orientation exist in the dynamical phase diagram, i.e. $\mathrm{SmA}_{\mathrm{I}}$ and $\mathrm{SmA}_{\mathrm{II}}$ [7]. In the $\mathrm{SmA}_{\mathrm{I}}$ region, both the parallel and the perpendicular orientations of the smectic layers shown in figure 1 coexist as originally suggested by Safinya et al [19]. On the other hand, the $\mathrm{SmA}_{\text {II }}$ phase is dominated by the perpendicular orientation in which the layer normal points along the vorticity direction $[19,20]$. The $\mathrm{SmA}_{\mathrm{I}}$ phase transforms into the $\mathrm{SmA}_{\mathrm{II}}$ phase upon increasing either shear stress or temperature. In this paper, we are mostly concerned with the $S m A_{I}$ regime, because the rheological property of the $\mathrm{SmA}_{\text {II }}$ phase is simply Newtonian due to the perpendicular orientation of the smectic layers.

\section{Experimental details}

We used the thermotropic liquid crystal 4-n-octyl-4'cyanobiphenyl $(8 \mathrm{CB})$ in the smectic phase. The equilibrium phase sequence and some physical properties of $8 \mathrm{CB}$ have been studied in the literature $[19,20]$. 8CB was obtained from Synthon Chemicals GmbH \& Co., Germany and was used without further purification. Rheological measurements were performed using the stress-controlled rheometer, Anton Paar MCR-300, equipped with a truncated cone-plate geometry whose diameter is $50 \mathrm{~mm}$ and the cone angle is $1^{\circ}$. In contrast to [4], no surface treatment has been performed in our experiment. The absence of the homeotropic alignment
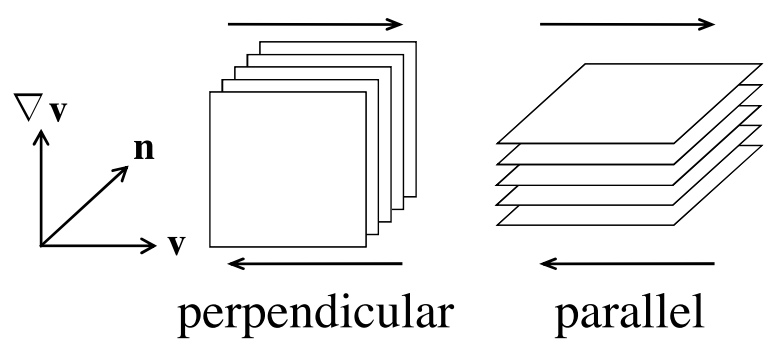

Figure 1. Schematic diagram of a smectic phase with perpendicular (left) and parallel (right) orientations under shear flow. $\nabla \mathbf{v}, \mathbf{v}$ and $\mathbf{n}$ correspond to the flow gradient, flow and vorticity directions, respectively. The $\mathrm{SmA}_{\mathrm{I}}$ region includes both the perpendicular and the parallel orientations, while only the perpendicular orientation appears in the $\mathrm{SmA}_{\text {II }}$ region [7].

of the smectic layers would easily induce the nucleation of focal parabolas under shear flow. However, we have checked that the reproducible results can be obtained by applying the preshear even without any surface treatment. As we will show in section 3 , our preshearing causes a similar effect to the anchoring treatment. Temperature was controlled within $0.02 \mathrm{~K}$ by a Peltier device attached to the rheometer. We determined the $\mathrm{SmA}-\mathrm{N}$ transition temperature $T_{\mathrm{SN}}$ by the dynamic viscoelastic measurement. From the condition that the storage modulus vanishes, we identified it as $T_{\mathrm{SN}}=$ $33.4^{\circ} \mathrm{C}$. This is in good agreement with the previously reported value [19]. The samples were presheared before the dynamic measurement at different shear stresses such that the FCD density was well controlled. Each shear stress was applied for $600 \mathrm{~s}$, which is long enough to reach the steady state.

Microscope observation under shear flow was performed by using the Linkam CSS-450 shear cell which has a plateplate shear geometry attached onto the Olympus microscope, BX-50, between the crossed polarizers. The gap size between the two plates was fixed at $150 \mu \mathrm{m}$. The microscope pictures were taken after applying the shear flow for $600 \mathrm{~s}$ with different fixed shear rates.

\section{Results}

\subsection{Scaling of shear modulus}

For each applied shear stress, the dynamic modulus was measured after the steady state shear rate was reached. The measured steady state shear rate $\dot{\gamma}$ as a function of the applied shear stress $\sigma$ is shown in figure 2. These data quantitatively reproduce our previous rheological measurement [7]. Most of the flow curves exhibit the shear thinning behavior. At high temperatures such as $33.2^{\circ} \mathrm{C}$, a dynamical transition from the shear thinning behavior to the Newtonian behavior is observed for large applied shear stresses. We note that the shear thinning and the Newtonian regimes correspond to the $\mathrm{SmA}_{\mathrm{I}}$ and the $\mathrm{SmA}_{\mathrm{II}}$ phases, respectively, as mentioned before. The data for $25^{\circ} \mathrm{C}$ almost coincide with those obtained by Horn and Kleman who performed a homeotropic anchoring treatment [4]. This implies that our preshearing treatment causes an analogous effect to the homeotropic anchoring 


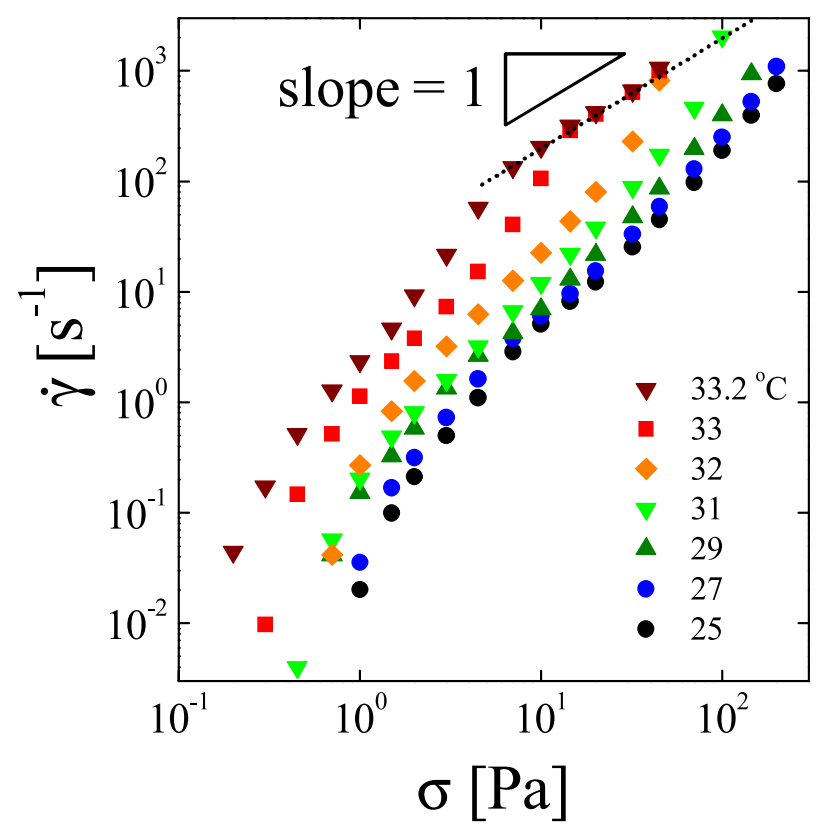

Figure 2. Steady state shear rate $\dot{\gamma}$ as a function of the applied shear stress $\sigma$ at different temperatures. The slope of the dotted line is unity, showing the Newtonian behavior $\dot{\gamma} \sim \sigma$.

treatment. Hereafter, we shall mainly discuss the shear modulus of the $\mathrm{SmA}_{\mathrm{I}}$ phase.

Figure 3 shows the frequency dependence of the dynamic storage modulus $G^{\prime}$ and the loss modulus $G^{\prime \prime}$ in the smectic phase at different temperatures. As reported before [5], we see that $8 \mathrm{CB}$ in the smectic phase generally shows a solidlike behavior since $G^{\prime}>G^{\prime \prime}$ for most of the frequencies measured. The extremely slow viscoelastic relaxation at the low frequency regime, indicative of the plastic behavior, can be attributed to the presence of defect structures. Indeed, Larson et al showed that the elimination of defects using large amplitude oscillatory shear diminishes both $G^{\prime}$ and $G^{\prime \prime}$ [3].

At each temperature, the plateau value of $G^{\prime}$ becomes larger as the preshear stress $\sigma$ increases, while $G^{\prime \prime}$ does not show any remarkable dependence on it. However, a further increase of $\sigma$ caused a reduction of $G^{\prime}$ and $G^{\prime \prime}$ as seen at $T=31$ and $33^{\circ} \mathrm{C}$ in figure 3 . The preshear dependence of $G^{\prime}$ can be attributed to the variation in the defect density which is regulated by the preshearing process $[3,4]$. We also find that the plateau value of $G^{\prime}$ decreases with increasing temperature. In particular, $G^{\prime}$ becomes remarkably smaller close to the Sm-N transition temperature $T_{\mathrm{SN}}$. Such a fall-off of $G^{\prime}$ by increasing the temperature is analogous to the decrease of the yield stress of the same smectic phase as reported in [7].

Figure 4 presents the dependence of the plateau value of the storage modulus $G^{\prime}$ at $\omega=0.1 \mathrm{~s}^{-1}$ on the shear rate $\dot{\gamma}$ and the reduced temperature $t=\left(T_{\mathrm{SN}}-T\right) / T_{\mathrm{SN}}$. (In the analysis, we used the absolute temperature so that $T_{\mathrm{SN}}=306.5 \mathrm{~K}$.) Adopting the result of figure 2, we use here the measured steady state shear rate $\dot{\gamma}$ for each applied preshear stress $\sigma$. To obtain the scaling plot of figure 4 , we first determined the power law behavior $G^{\prime} \sim \dot{\gamma}^{0.2}$ for each fixed temperature. Then we extracted the power law behavior of the scaled $G^{\prime}$ as a function of $t$ with an exponent of 0.7 so that all the data points

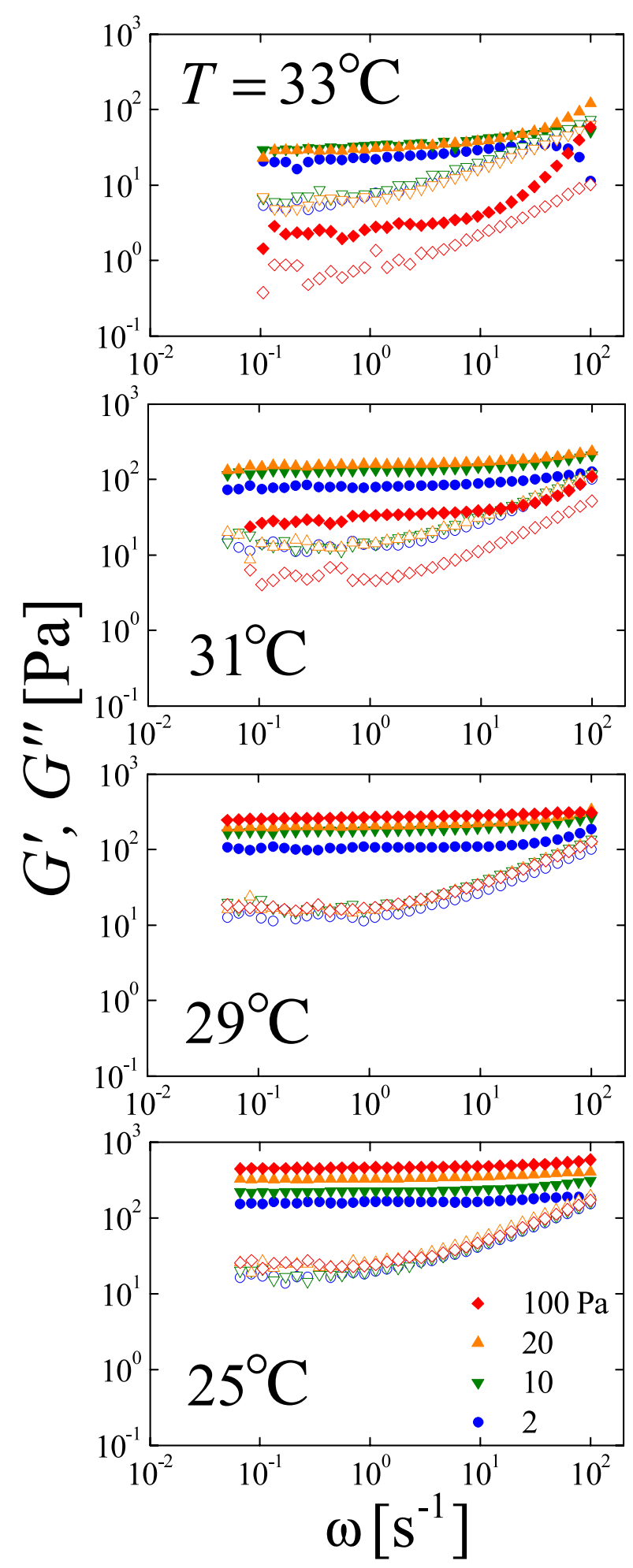

Figure 3. Dynamic storage modulus $G^{\prime}$ and loss modulus $G^{\prime \prime}$ as a function of frequency $\omega$ at different temperatures. Filled and open symbols correspond to $G^{\prime}$ and $G^{\prime \prime}$, respectively. Different symbols shown in the bottom panel represent the applied preshear stresses for which the steady states are obtained.

fall onto a single straight line whose slope is unity. After these procedures, we find the final scaling behavior $G^{\prime} \sim \dot{\gamma}^{0.2} t^{0.7}$. Notice that the slope of the dotted line in figure 4 is unity. The data collapse is satisfactory except for those at high preshear 


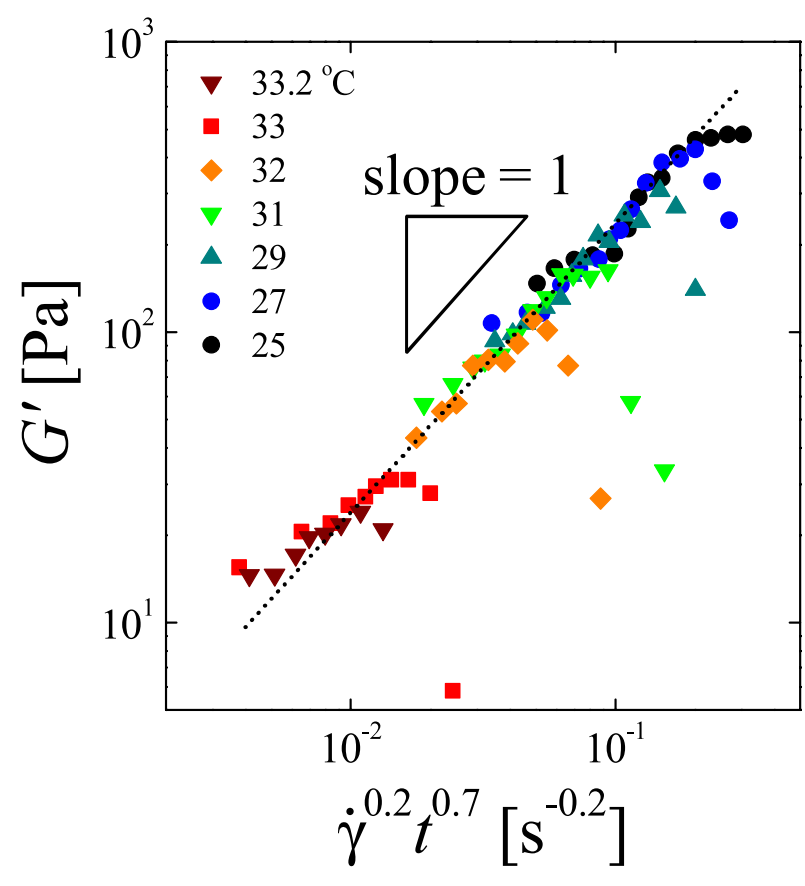

Figure 4. Plot of the plateau shear modulus $G^{\prime}$ as a function of the shear rate $\dot{\gamma}$ and the reduced temperature $t$. Different symbols correspond to different temperatures. The scaling variable is chosen to be $\dot{\gamma}^{0.2} t^{0.7}$ so that most of the data points fall onto a straight dotted line whose slope is unity.

stresses where $G^{\prime}$ decreases. The data which do not follow the scaling behavior indicate that they are close to the border between the $\mathrm{SmA}_{\mathrm{I}}$ and $\mathrm{SmA}_{\mathrm{II}}$ phases. This result indicates that the elasticity of the smectic phase is more enhanced as the shear rate (or the preshear stress) is increased and/or the temperature is reduced away from the transition temperature. The fact that $G^{\prime}$ can be scaled by the combined variable $\dot{\gamma}^{0.2} t^{0.7}$ means that both the shear rate and the proximity to the SN transition temperature effectively have the same influence on the elasticity of the smectic phase. For the onion phase in the surfactant lamellar phase, the dependence of the yield stress on the shear rate is also given by the power law behavior with the same exponent of 0.2 in spite of the different structures [21].

\subsection{Scaling of FCD size}

Here we discuss the real-space observation of the smectic textures under an optical microscope. Figure 5 presents polarized microscope images obtained after applying preshear at different temperatures for various fixed shear rates $\dot{\gamma}$. These microscope images were obtained by observing the sample along the flow gradient direction $\nabla \mathbf{v}$. In these images, the flow direction $\mathbf{v}$ and the vorticity direction $\mathbf{n}$ correspond to the longitudinal and the horizontal ones, respectively. These pictures show that the FCDs occupy most of the observed area. For each temperature, the FCDs are made smaller with increasing $\dot{\gamma}$. If one further raises $\dot{\gamma}$, the FCDs become too small to be observed under an optical microscope. For example, FCDs are invisible in the image of $T=25^{\circ} \mathrm{C}$ and $\dot{\gamma}=200 \mathrm{~s}^{-1}$. We consider that the shrinkage of the FCDs is related to the drop-off of $G^{\prime}$ at high preshear stresses in
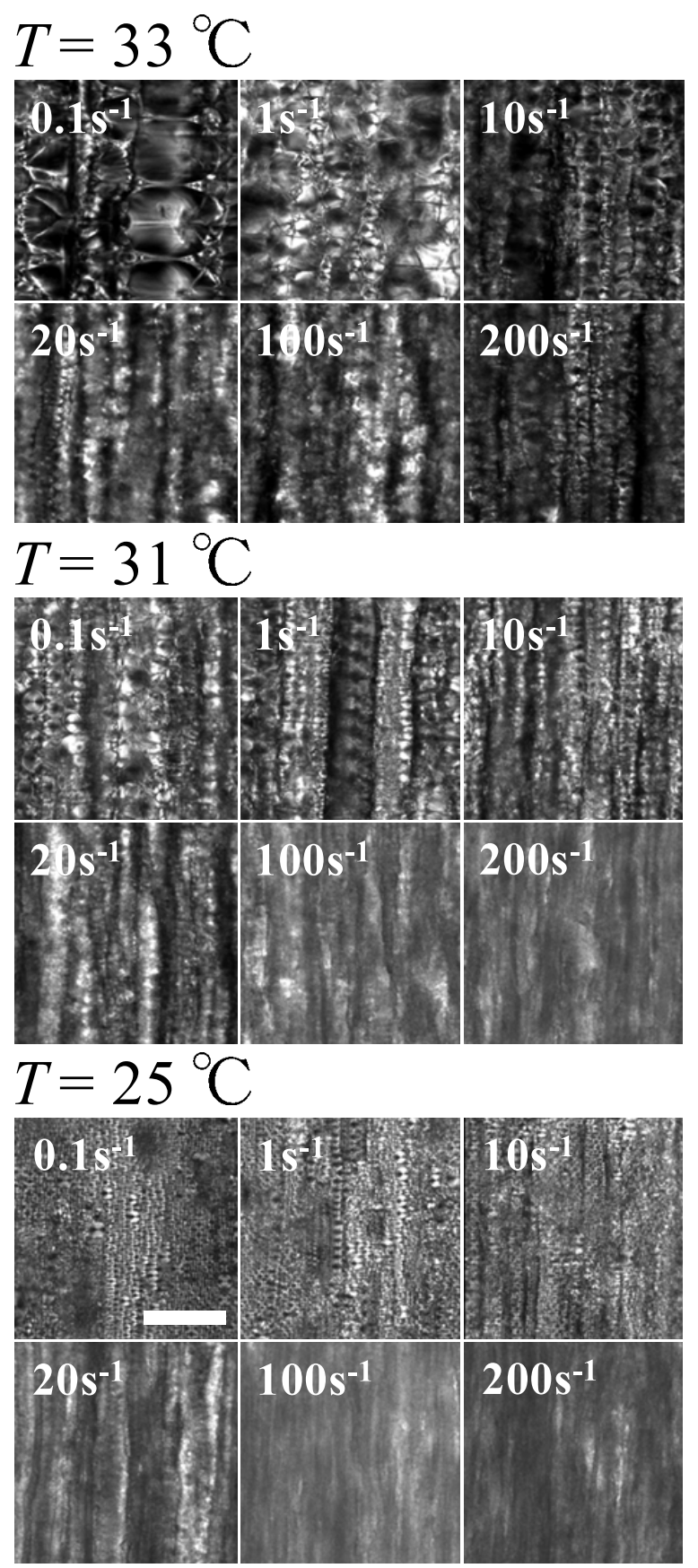

Figure 5. Polarized microscope images of the smectic phase under shear flow at different temperatures $\left(T=25,31\right.$ and $\left.33^{\circ} \mathrm{C}\right)$ and shear rates $\left(\dot{\gamma}=0.1,1,10,20,100\right.$ and $\left.200 \mathrm{~s}^{-1}\right)$. The flow is applied along the longitudinal direction. The scale bar corresponds to $100 \mu \mathrm{m}$.

figure 3. Hence the annihilation of FCDs in the microscope images and the deviation of $G^{\prime}$ from the dotted line in figure 4 is a signal for the onset of the parallel-to-perpendicular orientation transition. As for the temperature dependence, the FCD size remarkably increases with increasing temperature towards the transition temperature $T_{\mathrm{SN}}=33.4^{\circ} \mathrm{C}$. Such a trend can be clearly seen by comparing the smectic textures at $\dot{\gamma}=0.1 \mathrm{~s}^{-1}$ for different temperatures.

A typical example to extract the FCD size is presented in figure 6 which is obtained at $T=25^{\circ} \mathrm{C}$ when the shear rate is $\dot{\gamma}=0.1 \mathrm{~s}^{-1}$. As shown in figure 6 , the outlines of the FCDs 


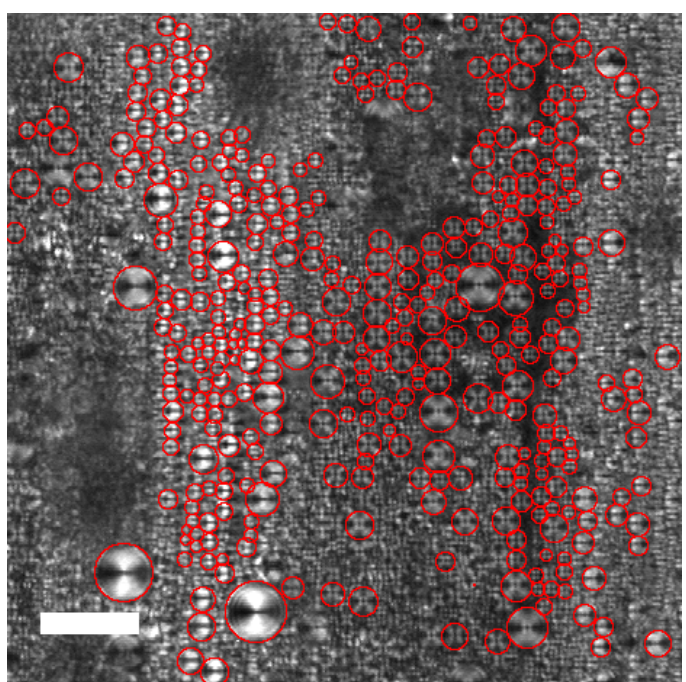

Figure 6. Typical microscope image used to estimate the FCD size at $T=25^{\circ} \mathrm{C}$ and $\dot{\gamma}=0.1 \mathrm{~s}^{-1}$. The averaged diameter $L$ of FCDs was estimated by tracing each FCD with a red circle as shown in the picture. The scale bar corresponds to $50 \mu \mathrm{m}$.

were traced with circles on the microscope images. Applying the same procedure to several microscope images obtained under the same condition, we extracted the averaged diameter $L$ for the given temperature and the shear rate. Although the FCD size obeys the distribution of the random Apollonian packing [22], we have extracted the averaged diameter $L$ by ignoring rather small FCDs. However, our result is essentially independent of the way we evaluate the average FCD size, besides some numerical factors.

In figure 7, we summarize the shear rate and the temperature dependence of the average FCD size. Here the upper and lower limits of the error bars correspond to the maximum and minimum size of the FCD, respectively. Our analysis revealed that $L$ obeys the power law behaviors on the shear rate $\dot{\gamma}$ as $L \sim \dot{\gamma}^{-0.2}$, and on the reduced temperature $t$ as $L \sim t^{-0.5}$. Hence the average FCD size can be well scaled by the combined variable $\dot{\gamma}^{-0.2} t^{-0.5}$ as verified in figure 7 . Here the data collapse onto a single line of slope unity is clearly demonstrated so that we can deduce the scaling relation $L \sim \dot{\gamma}^{-0.2} t^{-0.5}$. To the best of our knowledge, there is no model which describes the shear rate dependence of the FCD size. However, it is worthwhile to point out that a similar scaling relation $\xi \sim \dot{\gamma}^{-1 / 5}$ was predicted between the defect spacing $\xi$ and the shear rate $\dot{\gamma}$ as mentioned in section $1[14,15]$. Generally, FCDs can form strings connected by edge dislocations [23], while a dislocation loop consists of pairs of screw and edge dislocations. Although the topological relation between FCDs and dislocation loops is unknown, the same exponent of 0.2 may arise provided that the scale of edge dislocations regulates the FCD size.

Regarding the temperature dependence, the following discussions are in order. According to the defect model of the SmA-N transition proposed by Helfrich [24], smectic layers are destroyed by a proliferation of dislocation loops as the transition point is approached from below. He predicted that the average size of the dislocation loops diverges at the SmA$\mathrm{N}$ transition point as $\xi \sim t^{-0.5}$. Such a melting behavior of the

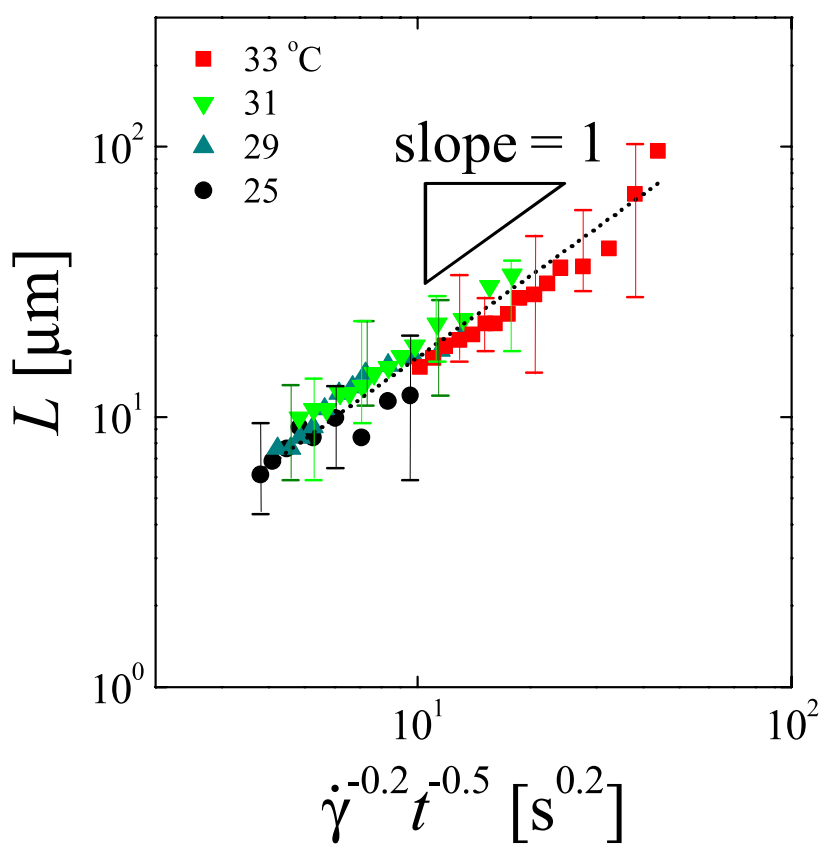

Figure 7. Plot of the FCD size $L$ as a function of the shear rate $\dot{\gamma}$ and the reduced temperature $t$. Different symbols correspond to different temperatures. The scaling variable is chosen to be $\dot{\gamma}^{-0.2} t^{-0.5}$ so that all the data points fall onto a straight dotted line whose slope is unity.

smectic order was experimentally confirmed in the vicinity of the smectic-cholesteric transition for a chiral system [25]. If we assume that there exists only one characteristic length scale close to the critical temperature [18], $\xi$ should be proportional to the FCD size $L$, and hence the obtained relation $L \sim t^{-0.5}$ is reasonable.

\subsection{Origin of elasticity}

By conducting independent measurements, we have experimentally obtained the scaling relation for the shear modulus as $G^{\prime} \sim \dot{\gamma}^{0.2} t^{0.7}$ and that for the average FCD size as $L \sim$ $\dot{\gamma}^{-0.2} t^{-0.5}$. These two relations strongly lead us to suggest that $G^{\prime}$ is inversely proportional to $L$, although this seems to be not completely correct because of the slight difference in the temperature exponents $(0.7$ versus 0.5$)$. However, this inconsistency can be resolved if the proportionality coefficient would also depend on the temperature. We show below that this is indeed the case.

If we assume that the relation $G^{\prime} \sim 1 / L$ holds, the proportionality coefficient on the right-hand side should have the dimension of surface tension, i.e. energy per area. De Gennes pointed out that the layered system such as the smectic phase or lamellar phase indeed exhibits surface tension [18]. According to [18, 26, 27], the effective surface tension of the layered system is given by $\gamma_{\mathrm{eff}}=\sqrt{K B}$, where $K$ and $B$ are the bending modulus and the compression modulus of the smectic phase, respectively. We then propose the following relation:

$$
G^{\prime}=C \frac{\sqrt{K B}}{L}
$$

where $C$ is a dimensionless numerical coefficient. From the previous experimental papers, it is known that $K$ for $8 \mathrm{CB}$ is 
almost constant, $K=(5.2 \pm 0.3) \times 10^{-12} \mathrm{~N}$ [28], while $B$ depends on the temperature close to $T_{\mathrm{SN}}$, obeying the power law relation $B=\left(7.5 \times 10^{7}\right) t^{0.4 \pm 0.03} \mathrm{~Pa}$ [8]. With this relation, $B$ vanishes at the transition point $(t=0)$ because the SmA$\mathrm{N}$ transition is associated with the disruption of the layered structures.

In order to check the validity of (1), we compare the values of $G^{\prime} L$ obtained from our experiment and $\gamma_{\mathrm{eff}}=\sqrt{K B}$ estimated from the literature. By taking into account the prefactors in our previous scaling relations, we obtain $G^{\prime} L=$ $\left(4.56 \times 10^{-3}\right) t^{0.2} \mathrm{~N} \mathrm{~m}^{-1}$. On the other hand, the above values from the literature yield $\sqrt{K B}=\left(1.97 \times 10^{-2}\right) t^{0.2} \mathrm{~N} \mathrm{~m}^{-1}$. Hence (1) is totally valid in terms of the scaling with respect to both $\dot{\gamma}$ and $t$. Moreover, the dimensionless numerical factor can be determined to be $C=0.456 / 1.97 \approx 0.23$. The consistency of (1) demonstrates that the physical origin of the elasticity in the smectic phase is due to the effective surface tension $\gamma_{\text {eff }}$ of the FCDs. This is the main claim of the present paper.

Princen et al showed experimentally that the physical origin of the shear modulus and the yield stress in emulsions can be commonly attributed to the surface tension, although the volume fraction dependences of the shear modulus and the yield stress are different from each other [29, 30]. The fact that the above prefactor $C$ is smaller compared to that of monodispersed emulsions can be attributed to the polydispersity of the FCD size [29, 30].

\section{Discussion}

We first discuss the generality of (1). In fact, essentially the same relation was proposed for the shear-induced onion phase in surfactant solutions [31]. They experimentally found that the shear modulus of the onion phase follows the relation $G^{\prime}=C_{0}+C_{1} \sqrt{K B} / L$. Here the first constant term $C_{0}$ reflects the residual strain due to the disordered arrangement of the onion structure. The second term is the stored energy per unit volume and the quantity $\sqrt{K B}$ corresponds to the effective surface tension of a single onion under small deformation $[26,27]$. Hence they concluded that the elasticity of the onion phase originates in the energetic penalty associated with the onion deformation. The later experimental work reported that $C_{0}$ tends to vanish for the disordered onion systems, while the prefactor $C_{1}$ varies from 0.4 to 1.2 for different surfactants [32]. These results are in accord with our result for the smectic phase with FCDs.

It is well known that the structure of FCDs is classified into two types; one is the toroidally deformed FCD (FCDI) commonly observed in the thermotropic smectics and the other is the onion type (FCD-II) seen in the lyotropic liquid crystals [2]. In spite of the topological difference between the two types, our result suggests that the energy cost for the deformation is determined by $\sqrt{K B}$ both for FCD-I and FCDII. Both in the smectic phase and the surfactant onion phase, the main source of the elasticity seems to be the effective surface tension. Therefore the rheological response of these systems is generally described by the scaling relation of (1), which is universal.
As mentioned before, Horn and Kleman suggested a scaling relation $\sigma_{\mathrm{y}} \sim K / L^{2}$ for the yield stress of the smectic phase with FCDs [4]. This relation was also used in our previous paper in order to estimate the FCD size close to the transition temperature [7]. (A similar relation also holds even for the elasticity due to the line defects when $K$ and $L$ are replaced by the line tension and the defect spacing, respectively, because the elasticity of the defect network is proportional to the yield stress $[11,12]$.) We emphasize that our result (1) differs from it. We now speculate that the elasticity of the layered structures with FCDs should be properly accounted for by both $K$ and $B$. In particular, the layer compression modulus $B$ reflects the overall interactions between the adjacent layers.

It should be noted that the maximum size of the FCD at $\dot{\gamma}=0.1 \mathrm{~s}^{-1}$ and $T=33^{\circ} \mathrm{C}$ is about $100 \mu \mathrm{m}$, which is smaller than the gap size $150 \mu \mathrm{m}$ of the plate-plate cell. Hence we consider that the effect of the gap size on the FCD size is almost negligible. For the viscoelastic measurement in which the gap size changes from 50 to $436 \mu \mathrm{m}$, the FCDs at higher temperatures and lower shear rates would be compressed close to the center of the cone. However, the volume of such a compressed FCD region is negligibly small compared with the total volume of the sample. We thus believe that the gap size effect on the FCD size will not produce any significant influence on the shear modulus.

In our analysis, we have used the experimentally observed scaling $B \sim t^{0.4}[8]$. However, the real critical behavior of $B$ requires a more careful discussion because it was reported later that $B$ does not necessarily vanish at the transition point [9]. This result supports well the Nelson-Toner model of the SmA$\mathrm{N}$ transition with full anisotropy [10]. In our experiment, the temperature is not very close to the critical temperature so that the above relation is practically useful. On the other hand, a caution is required when we use this relation beyond the critical region at low temperature.

\section{Conclusion}

In summary, we have studied the viscoelastic properties of thermotropic smectic liquid crystals with FCDs. By measuring the shear modulus $G^{\prime}$ and the FCD size $L$ independently, we have found the scaling relations $G^{\prime} \sim \dot{\gamma}^{0.2} t^{0.7}$ and $L \sim$ $\dot{\gamma}^{-0.2} t^{-0.5}$. The product $G^{\prime} L \sim t^{0.2}$ scales in the same manner as the effective surface tension $\gamma_{\text {eff }}=\sqrt{K B} \sim t^{0.2}$. Our experimental finding suggests that the shear modulus is inversely proportional to the FCD size and the proportionality coefficient is set by the effective surface tension $\gamma_{\text {eff }}$. Hence we conclude that the physical origin of the elasticity in the smectic phase is attributed to the effective surface tension of the FCD. The similarity of the rheological response between the smectic phase and the surfactant onion phase points toward the universal behavior of the layered systems under nonequilibrium conditions.

\section{Acknowledgments}

We acknowledge useful discussions with $\mathrm{R} \mathrm{H}$ Colby. We thank $\mathrm{T}$ Takahashi and M Imai for allowing us to use the 
rheometer MCR-300 and the Linkam shear cell CSS-450, respectively. SF and SK acknowledge support by KAKENHI (Grant-in-Aid for Scientific Research) on Priority Areas 'Soft Matter Physics' and grant no. 21540420 from the Ministry of Education, Culture, Sports, Science and Technology of Japan.

\section{References}

[1] Larson R G 1999 The Structure and Rheology of Complex Fluids (Oxford: Oxford University Press)

[2] Oswald P and Pieranski P 2006 Smectic and Columnar Liquid Crystals (Boca Raton, FL: Taylor and Francis)

[3] Larson R G, Winey K I, Patel S S, Watanabe H and Bruinsma R 1993 The rheology of layered liquids: lamellar block copolymers and smectic liquid crystals Rheol. Acta 32245

[4] Horn R G and Kleman M 1978 Observations on shear-induced textures and rheology of a smectic-A phase Ann. Phys. 3229

[5] Colby R H, Ober C K, Gillmor J R, Connelly R W, Duong T, Galli G and Laus M 1997 Smectic rheology Rheol. Acta 36498

[6] Colby R H, Nentwich L M, Clingman S R and Ober C K 2001 Defect-mediated creep of structured materials Europhys. Lett. 54269

[7] Fujii S, Ishii Y, Komura S and Lu C-Y D 2010 Smectic rheology close to the smectic-nematic transition Europhys. Lett. 9064001

[8] Benzekri M, Marcerou J P, Nguyen H T and Rouillon J C 1990 Critical behavior of the layer compressional elastic constant $\mathrm{B}$ at the smectic-A-nematic phase transition Phys. Rev. B 419032

[9] Benzekri M, Claverie T, Marcerou J P and Rouillon J C 1992 Nonvanishing of the layer compressional elastic constant at the smectic-A-to-nematic phase transition: a consequence of Landau-Peierls instability? Phys. Rev. Lett. 682480

[10] Nelson D R and Toner J 1981 Bond-orientational order, dislocation loops, and melting of solids and smectic-a liquid crystals Phys. Rev. B 24363

[11] Basappa G, Suneel Kumaran V, Nott P R, Ramaswamy S, Naik V M and Rout D 1999 Structure and rheology of the defect-gel states of pure and particle-dispersed lyotropic lamellar phases Eur. Phys. J. B 12269

[12] Ramos L, Zapotocky M, Lubensky T C and Weitz D A 2002 Rheology of defect networks in cholesteric liquid crystals Phys. Rev. E 66031711

[13] Bandyopadhyay R, Liang D, Colby R H, Harden J L and Leheny R L 2005 Enhanced elasticity and soft glassy rheology of a smectic in a random porous environment Phys. Rev. Lett. 94107801

[14] Meyer C, Asnacios S, Bourgaux C and Kleman M 2000 Rheology of lyotropic and thermotropic lamellar phase Rheol. Acta 39223
[15] Meyer C, Asnacios S and Kleman M 2001 Universal properties of lamellar systems under weak shear Eur. Phys. J. E 6245

[16] Orowan E 1940 Problems of plastic gliding Proc. Phys. Soc. $\mathbf{5 2} 81$

[17] Lu C-Y D, Chen P, Ishii Y, Komura S and Kato T 2008 Non-linear rheology of lamellar liquid crystals Eur. Phys. J. E 2591

[18] de Gennes P G and Prost J 1993 The Physics of Liquid Crystals (London: Clarendon)

[19] Safinya C R, Sirota E B and Plano R J 1991 Nematic to smectic-A phase transition under shear flow: a nonequilibrium synchrotron x-ray study Phys. Rev. Lett. 661986

[20] Panizza P, Archambault P and Roux D 1995 Effects of shear on the smectic A phase of thermotropic liquidcrystals J. Physique II 5303

[21] Fujii S and Richtering W 2006 Size and viscoelasticity of spatially confined multilamellar vesicles Eur. Phys. J. E 19139

[22] Ramos L, Roux D, Olmsted P D and Cates M E 2004 Equilibrium onions? Europhys. Lett. 66888

[23] Boltenhagen P, Lavrentvich O and Kleman M 1991 Oily streaks and focal conic domains in $L_{\alpha}$ lyotropic liquidcrystals J. Physique II 11233

[24] Helfrich W 1978 Defect model of the smectic A-nematic phase transition J. Physique 391199

[25] Moreau P, Navailles L, Giermanska-Kahn J, Mondain-Monval O, Nallet F and Roux D 2006 Dislocation-loop-mediated smectic melting Europhys. Lett. 7349

[26] van der Linden E and Droege J H M 1993 Deformability of lamellar droplets Physica A $\mathbf{1 9 3} 439$

[27] van der Linden E, Hogervorst W T and Lekkerkerker H N W 1996 Relation between the size of lamellar droplets in onion phases and their effective surface tension Langmuir 123127

[28] Zywocinski A, Picano F, Oswald P and Geminard J C 2000 Edge dislocation in a vertical smectic - a film: line tension versus temperature and film thickness near the nematic phase Phys. Rev. E 628133

[29] Princen H M and Kiss A D 1986 Rheology of foams and highly concentrated emulsions III. Static shear modulus J. Colloid Interface Sci. 112427

[30] Princen H M and Kiss A D 1989 Rheology of foams and highly concentrated emulsions IV. An experimental study of the shear viscosity and yield stress of concentrated emulsions J. Colloid Interface Sci. 128176

[31] Panizza P, Roux D, Vuillaume V, Lu C-Y D and Cates M 1996 Viscoelasticity of the onion phase Langmuir 12248

[32] Leng J, Nallet F and Roux D 2001 Anomalous elasticity of an ordered lamellar liquid foam Eur. Phys. J. E 4337 